\section{A freight train dispatching approach for handling perturbations: a real case for the longest heavy-haul railway in China}

Freight train dispatching approach

\author{
Yaqin Zhang
}

Institute of Computing Technology,

China Academy of Railway Sciences Corporation Limited, Beïing, China

Mingming Wang

School of Traffic and Transportation, State Key Laboratory of Rail Traffic Control and Safety, Beijing Jiaotong University, Beijing, China, and

Ruimin Wang, Zhipeng Li and Nan Zhang

Institute of Computing Technology,

China Academy of Railway Sciences Corporation Limited, Beijing, China
Received 29 September 2020 Revised 16 October 2020 Accepted 16 October 2020

\begin{abstract}
Purpose - This paper aims to reschedule the freight train timetable in case of disturbance to restore the train services as soon as possible.

Design/methodology/approach - Hence, an integer linear programming model for the real-time freight heavy-haul railway traffic management is developed in case of large primary delays caused by the delayed cargos loading. The proposed model based on the alternative graph at the microscopic level depicts the freight train movements in detail. Multiple dispatching measures such as re-timing and re-ordering are taken into account. Moreover, two objective functions, namely, the total final delays and the consecutive delays, are minimized in the freight trains dispatching problem.

Findings - Finally, a real-world computational experiment based on the Haolebaoji-Ji'an freight heavy-haul railway is implemented. The results of all disrupted cases are obtained within $10 \mathrm{~s}$. The results give insight into that the consecutive delays are more than the total final delays when the same disrupted situation and the consecutive or total final delays increase as the primary delays increase.

Originality/value - An integer linear programming model based on the alternative graph for the real-time freight heavy-haul railway traffic management is developed in case of large primary delays caused by the delayed cargos loading. The method can be developed as the computer-aided tool for freight train dispatchers.
\end{abstract}

Keywords Integer linear programming, Freight heavy-haul railway, Train dispatching problem

Paper type Research paper

(c) Yaqin Zhang, Mingming Wang, Ruimin Wang, Zhipeng Li and Nan Zhang. Published in Smart and Resilient Transportation. Published by Emerald Publishing Limited. This article is published under the Creative Commons Attribution (CC BY 4.0) licence. Anyone may reproduce, distribute, translate and create derivative works of this article (for both commercial and non-commercial purposes), subject to full attribution to the original publication and authors. The full terms of this licence maybe seen at http://creativecommons.org/licences/by/4.0/legalcode

This research was supported by the foundation project of China Academy of Railway Sciences Corporation Limited (2019YJ121).

This paper forms part of special section "Intelligent Technology and Application of Freight Railway", guest edited by Jiansheng Zhu, Ruimin Wang and Peng Xie.

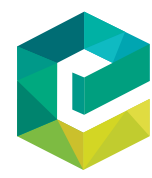

Smart and Resilient Transportation Vol. 2 No. 2, 2020 pp. 85-99 Emerald Publishing Limited e-ISSN: 2632-049 p-ISSN: $2632-048$ DOI 10.1108/SRT-09-2020-0009 
SRT

2,2

86

\section{Introduction}

The freight railway industry has been essential to society and economy, as it transports the strategic goods such as grain, coal and ore. Since then, the percentage of freight hauled by railroads has increased continuously. Thus, the efficient freight heavy-haul timetable is critical to freight railway system. In the freight heavy-haul trains operation process, disturbances are unavoidable such as primary departure delays owing to delayed cargos loading, the serious weather conditions and infrastructure failures. Therefore, the real-time and high-efficient disposition timetable is significant for freight railway operators and dispatchers. Hence, an optimum real-time rescheduling timetable has to be obtained while the influence of disturbances is minimized.

Train movement can be described as the sequence of train arrival and departure at specific points in the railway lines. The railway network consists of stations and segments divided by signals. At the macroscopic level the stations and segments can be viewed as points and arcs, and the signals are ignored (Veelenturf et al., 2016). However, the railway network can be viewed as the set of block sections considered the signal points at the microscopic level (D'Ariano et al., 2007a, D'Ariano et al., 2007b). Based on description of the railway network, the train movement can be illustrated at the macroscopic or microscopic level. When disturbances occur, the train movements can be rescheduled in practice to restore the freight train services.

In this paper, a real-time freight heavy-haul train-rescheduling problem is addressed. In the context of the passenger train dispatching problem, a lot of recover models and algorithms have been developed (Cacchiani et al., 2014; Corman and Meng, 2015). The freight heavy-haul railway system is similar to the high-speed railway system at the operation module when considering the trains need run on the special railway track conditions. Thus, some methods applied to passenger train dispatching problem have been considered in our paper.

Freight railroads system usually publishes their scheduled train timetable. When disruptions occur, trains movements are more likely to be disrupted, and the train conflicts occur. In our paper, we focus on the real-time train timetable rescheduling problem which detects train conflicts and obtain the optimum conflict resolution in real-time. We study the real-time freight heavy-haul train traffic management at the macroscopic level with considering in high-level constraints and disregarding detailed information on routes inside stations or junctions.

The main contributions of this paper consist of threefold. First, an integer linear programming model for real-time freight heavy-haul train timetable rescheduling problem is developed in case of disrupted situations. Our second contribution is related to dispatching measures. Re-timing and re-ordering are integrated into this model and two objective functions:

(1) the minimization of the total final delays; and

(2) the minimization of the consecutive delays are considered in the proposed model.

Third, a real-world experiment based on the Haolebaoji-Ji'an freight heavy-haul railway is implemented to test our developed model. The results of all disrupted cases are obtained in real-time. Moreover, the results give insight into that the consecutive delays are more than the total final delays when the same disrupted situation, and the consecutive or total final delays increase as the primary delays increase.

The paper is organized as follows. A literature review related to the train-dispatching problem is presented in Section 2. The problem description and an integer linear programming 
are elaborated in Section 3 and Section 4. Section 5 reports on our computational experiment. Finally, the conclusion and further work is discussed in Section 6.

\section{Literature review}

In this section we focus on the progress of freight heavy-haul railway dispatching methods. The train-dispatching problem has been a classical issue and received increased attention (Cordeau et al., 1998). The train-dispatching problem can be viewed as several problems, such as train conflicts detection problem and conflicts resolution problem (Corman et al., 2010). More detailed recover models and algorithms are developed for the passenger railway system. However, these models can also be used after they are adjusted according to the characteristic of the freight heavy-haul railway system.

The main task of train dispatching model is to determine where trains conflicts will occur and how to address the trains conflicts to minimize train delays or deviations from the scheduled timetable. When two or more trains require the same operation resources at the same time, the trains conflicts occur. The trains conflicts detection method is usually used the blocking time theory (Pranzo, 2004). This theory takes the signaling and safety aspects into account, and the railway network is divided into block section at the microscopic level. The popular microscopic formulation modeling the railway network is alternative graph (D'Ariano and Pranzo, 2009; D'Ariano et al., 2008). Each train movement in block section is defined as an operation. Each operation has its start time and operating time duration. A dispatching computer-aided tool called railway traffic optimization by means of alternative graph is also developed based on the alternative graph (D'Ariano et al., 2007a, Quaglietta et al., 2016). In our paper, we also develop a microscopic model taking detailed signaling points and routes inside stations.

For the train conflicts resolution problem, several dispatching measures such as retiming, re-ordering and re-routing are considered in the real-time railway traffic management. In the different disrupted situations, the proper dispatching strategies are applied. For the primary delays, most literature adjusts the trains' departure time or arrival time at stations, and changes trains' order to tackle the train conflicts (Corman et al., 2017). In the dispatching process, the station capacity some studies also considered in cases of small disturbances (Dollevoet et al., 2015). Furthermore, other constraints, for example, connection constraints, are also considered into their proposed model to minimize the inconvenience of passengers (Dollevoet et al., 2012). However, this context is beyond the scope of our researched real-time freight heavy-haul railway traffic management.

In recent studies the goals train dispatching model can be categorized as the train delays, the cost of operation and the convenience of passengers (Binder et al., 2017). For the train delays the authors make a distinction between the total final delays and the consecutive delays. The cost of operation and the convenience of passengers are also based on the train rescheduled timetable structure (Corman et al., 2011). Thus, in our proposed model the total final delays and the consecutive delays are set as the objective functions. In the real-time freight heavy-haul railway traffic management, the train final delays are weighted calculated in the range of acceptable arrival time window. Hence the objective functions proposed in this paper are distinct from other previous literature.

To summarize, in the current studies the more authors focus on the passenger trains dispatching problem. However, the real-time freight heavy-haul railway traffic management
Freight train dispatching approach 
SRT

2,2

is also important for the railway management departments. As its constraints differ quite significantly from passenger train dispatching problem, in current paper an extended freight heavy-haul train dispatching mathematical formulations is developed as integer linear optimization problem.

\section{Problem description}

A railway network is composed of sections and signals and can be divided into several sections by adjacent signals at the same directions. The section between the adjacent signals of the same direction is named as block section. Signals are allocated in the entrance of block sections and control the occupation of block sections with restrained speed level. The train passes through a block section is named operation. The operation can be classified as running operation, dwell operation and through operation. Each operation has specific its duration.

To guarantee safety of trains movement, a block only can host one train at the same time. Thus, a situation where two or more trains need to occupy the same block is defined as a train conflict. Thus, the train conflict detection and resolution problem is defined as that dispatchers find out the train conflicts and determine the orders of trains passing though the points where the train conflicts occur.

Let the operation be defined as node, and the connection of consecutive nodes be defined as fixed arc. The possible orders of trains at the conflict point is defined as alternative arcs. Thus, the $(G=N, F, A)$ denotes that the alternative graph, where the $N$ denotes the set of the operations, $F$ presents the set of the fixed arcs, and $A$ means the set of the alternative arcs. More information about the alternative graph we refer to can be found in (D'Ariano et al., 2007a).

Given a simple railway network for example, Figure 1 illustrates a simple railway network. The railway network is divided as 8 block sections by signals. There exist three trains $t_{1}, t_{2}$ and $t_{3}$ running on this railway network. To clarify the railway network clearly, we show the most relevant block sections in Figure 1.

From the scheduled timetable, the predefined route of train $t_{1}$ consists of operations $\left(t_{1}, 1\right)$, $\left(t_{1}, 3\right),\left(t_{1}, 4\right),\left(t_{1}, 5\right),\left(t_{1}, 6\right)$ and $\left(t_{1}, 7\right)$. The predefined route of train $t_{2}$ is the sequence of operations $\left(t_{2}, 1\right),\left(t_{2}, 3\right),\left(t_{2}, 4\right),\left(t_{2}, 5\right),\left(t_{2}, 6\right)$ and $\left(t_{2}, 8\right)$. The route of train $t_{3}$ is $\left(t_{3}, 2\right),\left(t_{3}, 3\right),\left(t_{3}, 4\right),\left(t_{3}, 5\right),\left(t_{3}, 6\right)$ and $\left(t_{3}, 8\right)$. Train $t_{1}$ and train $t_{2}$ share the same block sections $1,3,4,5$ and 6 . Train $t_{2}$ and train $t_{3}$ share the same block sections $3,4,5,6$ and 8 . Train $t_{1}$ and train $t_{3}$ share the same block sections 3, 4, 5 and 6. Two dummy nodes $o$ and $d$ which represent the start and end respectively are added into the alternative graph. The alternative graph of the simple railway network presented by Figure 1 is described by Figure 2. The solid arc represents the fixed arc, and the dashed arc illustrates the alternative graph. The alternative arcs are pairs, and only one of a pair existed feasible solutions.

In our paper, a heavy haul freight railway real-time traffic management problem is formulated based on the alternative graph. We do not re-route of trains and canceled trains. The re-timing and re-ordering dispatching measures are considered in the real-time traffic management in case of disrupted situations.

Figure 1.

Simple railway network

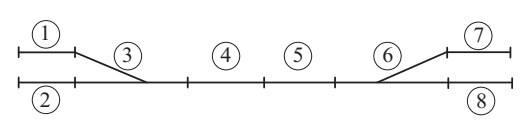




\section{Integer programming model}

In Section 4, the problem assumptions, parameters and decision variables list and real-time traffic management model for a heavy haul freight railway.

Freight train dispatching approach

\subsection{Problem assumptions}

Real-time timetable rescheduling problem in case of large-scale disrupted situations is a complexity. To clarify the problem researched in this paper, some assumptions is defined as follows:

- The route of each freight train is fixed, and it is predetermined by the scheduled timetable. Because the heavy haul freight train has its special route, the route of each freight train is not allowed to be changed.

- The characteristics of the disrupted situation is certain, such as disruption type, its start time, its duration time length and its position. When a disruption occurs, the disruption type, its start time and its position can be obtained from the infrastructure managers. Moreover, the duration time length of the disruption can be predicted by the prediction model formulated by historical data (Zilko et al., 2016).

- The rolling stock and freight carriages rescheduling phases are not considered in the context of our paper. The disruption management consists of the timetablerescheduling phase, rolling stock rescheduling phase and crew-rescheduling phase. The rescheduled train timetable is as the input of other phases and is an essential phase of the disruption management. A lot of literature related about the rolling stock and crew rescheduling assumes that the timetable is predetermined (Potthoff et al., 2010) (Lusby et al., 2017).

- The infrastructure characteristics, scheduled timetable, headway time, minimum operation time as the input of proposed model is obtained.

\subsection{Parameters and decision variables}

The notations of the proposed model are listed in Table 1.

\subsection{Real-time traffic management model}

In this section, a real-time traffic management model is formulated based on the alternative graph. The train movements and relations between adjacent trains can be descripted by the alternative graph. The constraints of a real-time traffic management model can be easily transformed.

4.3.1 Constraints for scheduled departure time. The freight train cannot depart earlier than the scheduled departure time plus the primary entrance delays, shown as constraints (1), as the scheduled departure time is the earliest time to complete freights loading and cargoes assembled which satisfies the conditions where the freight train is operated. If the

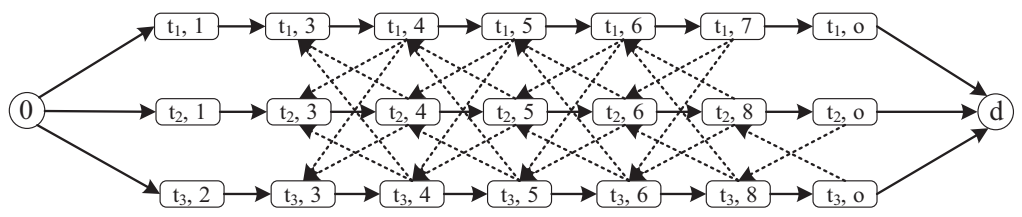

Figure 2.

Alternative graph for the railway network illustrated by Figure 1 


\begin{tabular}{|c|c|c|}
\hline SDT & & \\
\hline SK & Parameter & Description \\
\hline , & & The set of trains, $t r \in T$ \\
\hline & $B$ & The set of block sections, $i \in B$ \\
\hline & G & The alternative graph \\
\hline & $N$ & The set of nodes of the alternative graph $G$ \\
\hline & F & The set of fixed arcs of the alternative graph $G$ \\
\hline & $A$ & The set of alternative arcs of the alternative graph $G$ \\
\hline 90 & $R_{t r}$ & The route of train $t r$ \\
\hline & & The original block section of train $t r$ \\
\hline & $b_{d r}$ & The disrupted block section \\
\hline & $B_{t r}^{d w e l l}$ & The set of block sections where train $t r$ dwell \\
\hline & $B_{d i s}$ & The set of disrupted block sections \\
\hline & $T_{\text {dis }}^{\text {start }}$ & The start time of a disruption \\
\hline & $T_{d i s}^{\text {des }}$ & The end time of a disruption \\
\hline & $d t$ & The primary delays \\
\hline & $t_{i}$ & The operation time at block section $i$ \\
\hline & & The node adjacent to node $i$ \\
\hline & $((\sigma(i), j),(\sigma(j), i))$ & A pair of alternative $\operatorname{arcs},((\sigma(i), j),(\sigma(j), l)) \in A$ \\
\hline & $t_{(\sigma(i), j)}$ & The duration time of a block section occupied by adjacent train \\
\hline & $e_{(t r, i)}$ & The scheduled time of train $t r$ enters in block section $i$ \\
\hline & Decision variables & Descriptions \\
\hline & $\omega_{(t r, i)}$ & The actual time of train $t r$ enters in block section $i$ \\
\hline & $\chi_{(\sigma(i), j)}$ & The binary decision variable which if alternative $\operatorname{arc}(\sigma(i), j)$ is selected, \\
\hline $\begin{array}{l}\text { Table } 1 . \\
\text { Parameters list }\end{array}$ & $z_{t r}$ & $\begin{array}{l}\chi_{(\sigma(i), j)}=1 \text {; otherwise, } \chi_{(\sigma(i), j)}=0 \\
\text { The binary decision variable which if train } t r \text { is disrupted by a } \\
\text { disruption, } z_{t r}=1 \text {; otherwise, } z_{t r}=0\end{array}$ \\
\hline
\end{tabular}

freight train is not delayed, the rescheduled departure time from its original station only greater than its scheduled time, shown as constraints (2):

$$
\begin{gathered}
\omega_{\left(t r, b_{t r}^{o}\right)} \geq e_{\left(t r, b_{t r}^{o}\right)}+d t_{\left(t r, b_{t r}^{o}\right)}, t r \in T_{\text {delayed }} \\
\omega_{\left(t r, b_{t r}^{o}\right)} \geq e_{\left(t r, b_{t r}^{o}\right)}+d t_{\left(t r, b_{t r}^{o}\right)}, t r \in T \backslash T_{\text {delayed }}
\end{gathered}
$$

4.3.2 Constraints for minimum operation time. Each operation has its operation time, and the train must satisfy the minimum operation time. The operations can be divided into running and dwell operation considered the different operation type. And then the actual duration time of running and dwell operation is greater than the minimum running time and dwell time, respectively, shown as constraints (3)-(4):

$$
\begin{gathered}
\omega_{(t r, j)} \geq \omega_{(t r, i)}+t_{i}^{d w e l l, \min }, t r \in T, j=\sigma(i), i \in B_{t r}^{d w e l l} \\
\omega_{(t r, j)} \geq \omega_{(t r, i)}+t_{i}^{r m n, \min }, \operatorname{tr} \in T, j=\sigma(i), i \in R_{t r} \backslash B_{t r}^{d w e l l}
\end{gathered}
$$

4.3.3 Constraints for order of precedence. The order of precedence determines the optimum order of two trains which require the same block section at the same time. At the merging 
points, the actual start time of an operation is restricted by its precedential operation, shown as constraints (5)-(7):

$$
\begin{gathered}
\omega_{\left(t_{2}, j\right)} \geq \omega_{\left(t_{1}, \sigma(i)\right)}+t_{(\sigma(i), j)}+M \cdot \chi_{(\sigma(i), j)} \\
\omega_{\left(t r_{1}, i\right)} \geq \omega_{\left(t r_{2}, \sigma(j)\right)}+t_{(\sigma(j), i)}+M \cdot \chi_{(\sigma(j), i)} \\
\chi_{(\sigma(i), j)}+\chi_{(\sigma(j), i)}=1 \\
t r_{1} \neq t r_{2}, t r_{1}, t r_{2} \in T,((\sigma(i), j),(\sigma(j), i)) \in A
\end{gathered}
$$

The logistic order of precedence means one order can be determined from other related orders when two more trains require the same block section. Figure 3 illustrates a situation where three trains require the same block section. The red solid arcs represent the selected alternative, and the gray dotted arcs are not selected $\operatorname{arcs}$. When the alternative $\operatorname{arc} \chi_{(\sigma(i), j)}$ of train $t r_{1}$ and $t r_{2}$ is selected and the alternative arc $\chi_{(\sigma()), k)}$ of train $t r_{2}$ and $t r_{3}$ is selected, the alternative arc $\chi_{(\sigma(i), k)}$ of train $t r_{1}$ and train $t r_{3}$ must be selected. Therefore, the logistic order of precedence is shown as constraints (8):

$$
\begin{gathered}
\chi_{(\sigma(i), k)}+1 \geq \chi_{(\sigma(i), j)}+\chi_{(\sigma(j), k)} \\
((\sigma(i), j),(\sigma(j), i)),((\sigma(j), k),(\sigma(k), j)),((\sigma(i), k),(\sigma(k), i)) \in A
\end{gathered}
$$

4.3.4 Constraints for disruptions. When a disruption occurs, the characteristics of this disruption, such as the start time and the location of the disruption can be easily obtained from infrastructure managers. And we assume that the duration of the disruption is known. The trains that are influenced can be identified by the scheduled start time. We introduce two kinds of binary decision variables $y_{t r}^{\prime}$ and $y_{t r}^{\prime \prime}$, and their conditions are described as follows:

$$
y_{t r}^{\prime}=\left\{\begin{array}{ll}
1 & e(t r, i)>T_{d i s}^{s t a r t} \\
0 & \text { Otherwise }
\end{array} \text { and } y_{t r}^{\prime \prime}= \begin{cases}1 & e(t r, \sigma(i))<T_{d i s}^{e n d} \\
0 & \text { Otherwise }\end{cases}\right.
$$

Introducing the M method, the constraints (9) can be linearized as constraints (10) and (11). The trains that are impacted by the disrupted situation are identified by constraints (12):

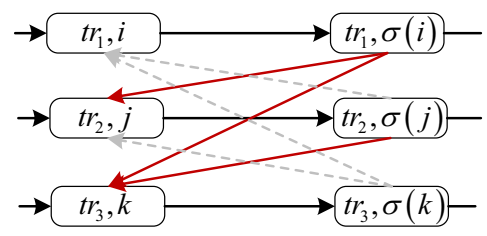

Figure 3. Situation where three train require the same block section 


$$
e(t r, i)-T_{d i s}^{s t a r t} \leq M \cdot y_{t r}^{\prime}, t r \in T, i \in R_{t r}
$$

$$
\begin{gathered}
e(t r, \sigma(i))-T_{d i s}^{e n d} \leq M \cdot y_{t r}^{\prime \prime}, t r \in T, \sigma(i) \in R_{t r} \\
z_{t r}+1 \geq y_{t r}^{\prime}+y_{t r}^{\prime \prime}, t r \in T
\end{gathered}
$$

When a block section is disrupted, the start time of train $t r$ enters this disrupted block section greater than end time of the disruption, shown as constraints (13):

$$
\omega(t r, i) \geq T_{d i s}^{e n d} \cdot z_{t r}, \operatorname{tr} \in T
$$

4.3.5 Objectives. Compared with passenger railway traffic system, the freight train is allowed to arrival at its destination station within a time horizon. The arrival time of freight trains are permitted to have a deviation $\delta$ from the scheduled arrival time at their destinations. When the deviation exceeds the minimum deviation $\delta_{\text {min }}$, this freight train results in large costs for the freight railway administration. Thus, the objective of the proposed model is as follows:

$$
f=\min \sum_{t r \in T} \max \left\{\left(\left|\omega\left(t r, b_{t r}^{d}\right)-e\left(t r, b_{t r}^{d}\right)\right|-\delta_{\min }\right), 0\right\}
$$

To linearize the objective function (14), we introduce two types of decision variables, $d_{t r}^{-}$and $d_{t r}^{+}$which mean the late or early time and the deviation time, respectively. A decision variable $d_{t r}$ denotes the maximum value of $d_{t r}-\delta_{\min }$ and 0 . The linear objective is as follows (15)-(20):

$$
\begin{gathered}
f=\min \sum_{t r \in T} d_{t r} \\
d_{t r}^{-}=\omega\left(t r, b_{t r}^{d}\right)-e\left(t r, b_{t r}^{d}\right) \\
d_{t r}^{+} \geq d_{t r}^{-} \\
d_{t r}^{+} \geq-d_{t r}^{-} \\
d_{t r} \geq d_{t r}^{+}-\delta_{\min } \\
d_{t r} \geq 0
\end{gathered}
$$

\section{Computational results}

In Section 5, we evaluate the performance of the proposed IP real-time freight railway traffic management model in a real-case study on Haolebaoji-Ji'an the longest haul freight railway in China. Section 5 is composed of the case description, parameter setting and results analysis. 


\subsection{Case description}

The Haolebaoji-Ji'an railway line is $1,806 \mathrm{~km}$ long which is the longest haul freight railway in China. There are in total 81 stations along this line, which divide the line into 80 segments in recently operation. The Haolebaoji-Jian railway line in China is shown in Figure 4. In our case study, we only consider 23 stations, from JingBianDong (JBD) to GouLinNan (GLN), are shown in the red circle in the Figure 4. These stations belong to the same dispatching area. The line is composed of 22 double-tracked segments from JBD to GLN.

\subsection{Parameters set}

For the real-time train timetable-rescheduling problem, the important parameters consist of the minimum running time, dwell time and the headway times. The minimum running times of sections are obtained from the statistical analysis of historical actual train timetables. The minimum dwell time of each train we assume is defined as the planned dwell time at its stop station. Based on the historical actual train timetables from October 1, 2019 to October 31, 2019, the actual running times are analyzed, shown as Figures (5 and 6). The minimum running time of each section we assume is defined as the minimization of its all historical running times. The minimum running time of each downside section is listed in Table 2, and each upside section is presented in Table 3. Moreover, we assume that the minimum dwell time of each train at its stop station is the scheduled dwell time in the original train timetable. The minimum headway time of the adjacent trains' arrival at or departure from the same station in sequence is defined as $900 \mathrm{~s}$. Because the Haolebaoji-Ji'an freight haul railway has only been in operation, the number of trains operated in daily is not dense. The scheduled timetable of October 25, 2019 shown as Figure 7 is used in all cases of the studied experiment.

\subsection{Disrupted scenarios}

Our experiment is based on different disrupted scenarios. The disrupted scenarios are depicted by the tuple of the disrupted train, location and the delays. The disrupted trains are selected randomly, and their delays are generated according to the uniform distribution with a range between 30 and $60 \mathrm{~min}$. The considered scenarios are as follows:

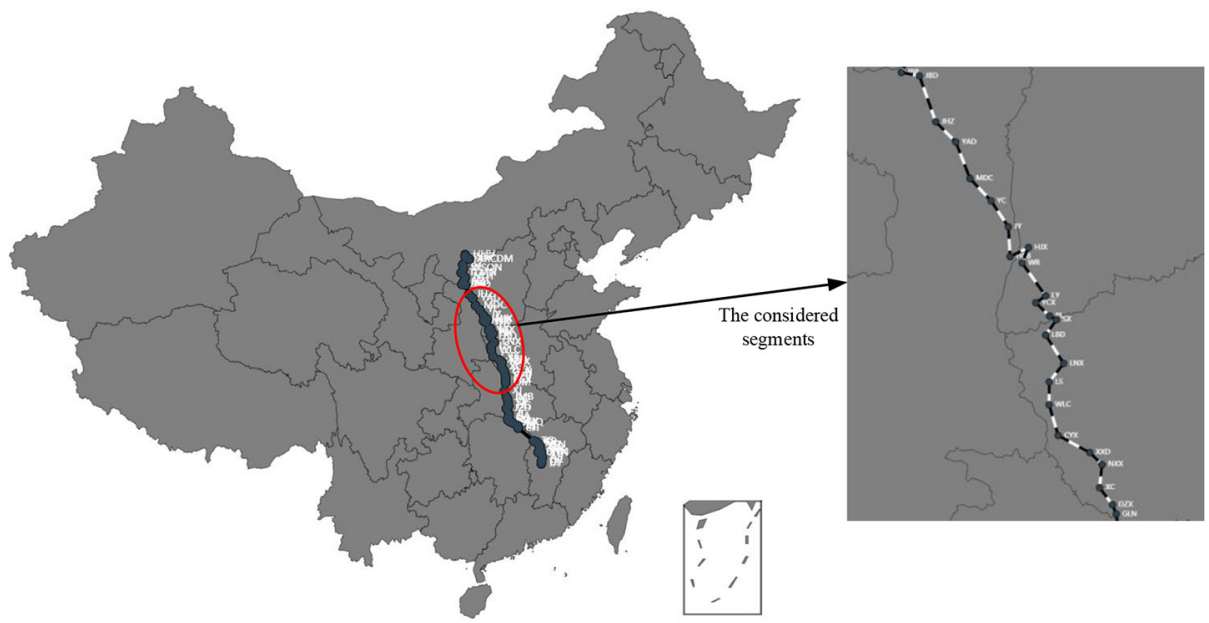

Freight train dispatching approach 

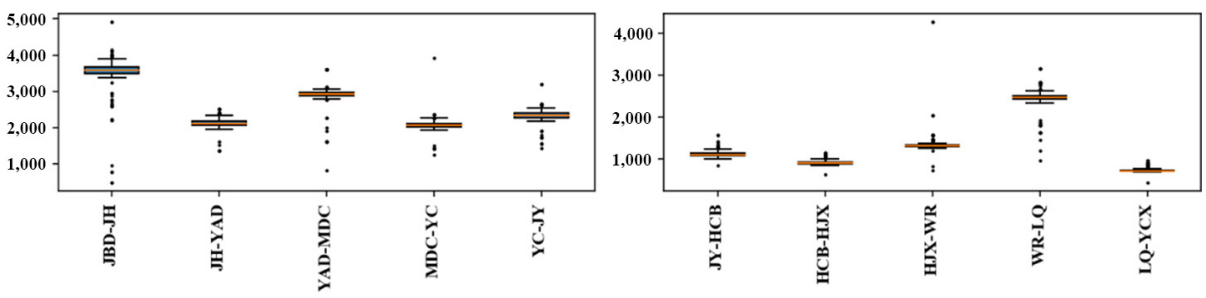

Figure 5.

Running time of downside sections
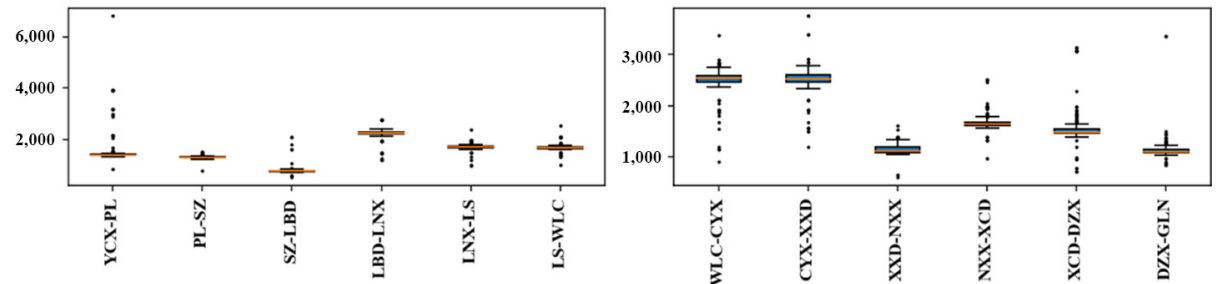

Table 2.

Minimum running time of each downside section

\begin{tabular}{lr}
\hline Section & Minimum runnin \\
\hline JBD-JH & 480 \\
JH-YAD & 1,380 \\
YAD-MDC & 840 \\
MDC-YC & 1,260 \\
YC-JY & 1,440 \\
JY-HCB & 845 \\
HCB-HJX & 620 \\
HJX-WR & 720 \\
WR-LQ & 960 \\
LQ-YCX & 421 \\
YCX-PL & 832
\end{tabular}

Section

PL-SZ

SZ-LBD

LBD-LNX

LNX-LS

LS-WLC

WLC-CYX

CYX-XXD

XXD-NXX

NXX-XCD

XCD-DZX

DZX-GLN
Minimum running time (s)

780

524

1,200

960

1,006

900

1,200

600

963

720

840

\begin{tabular}{lclr}
\hline Section & Minimum running time (s) & Section & Minimum running time (s) \\
\hline JH-JBD & 1,140 & SZ-PL & 787 \\
YAD-JH & 1,080 & LBD-SZ & 502 \\
MDC-YAD & 1,755 & LNX-LBD & 1,475 \\
YC-MDC & 1,272 & LS-LNX & 997 \\
JY-YC & 840 & WLC-LS & 1,003 \\
HCB-JY & 618 & CYX-WLC & 1,435 \\
HJX-HCB & 554 & XXD-CYX & 1,412 \\
WR-HJX & 841 & NXX-XXD & 644 \\
LQ-WR & 1,461 & XCD-NXX & 966 \\
YCX-LQ & 423 & DZX-XCD & 987 \\
PL-YCX & 883 & GLN-DZX & 840 \\
\hline
\end{tabular}



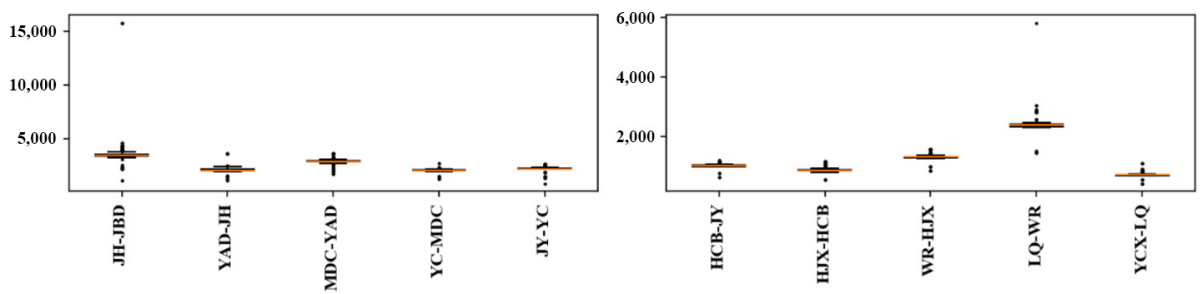

Freight train dispatching approach
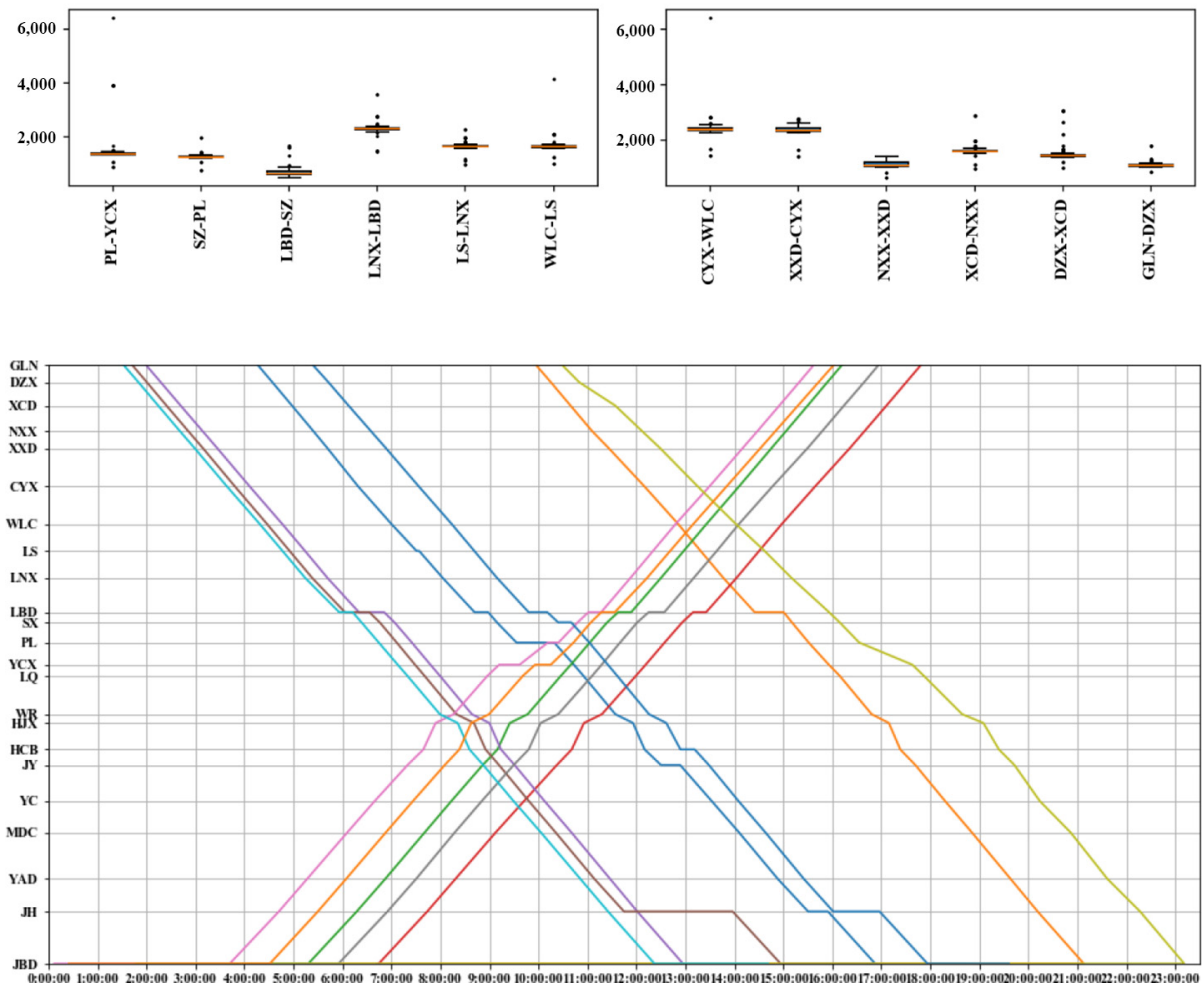

Figure 7.

Original train timetable used in all cases of the studied experiment

First, we assume that disrupted trains can be upside or downside. Because of the difference of the minimum running time of the different directions, the impact of the different durations of primary delays on the whole day timetable is analyzed.

Second, the different types of primary delays are considered such as arrival and departure primary delay and the entrance delays in the original station.

Finally, the influence of the different locations of the disruption is studied. Because the different station capacity is restricted, the disposition timetable is generated by different dispatching strategies.

In our experiment, a tuple of the disrupted train, occurrence location and delays $(T r, S$, $D T$ ) describes each disrupted scenario. Here $T r, S$ and $D T$ denote the set of the disrupted trains, their locations and the delays, respectively. The delays consist of the delay type and duration time (type, $d t)$. 
SRT

2,2

96

\subsection{Computational results}

In subsection 5.4, the computational results obtained by the proposed mothed are described as follows. The proposed method is implemented by commercial solver Gurobi 9.0 and Python on a Window 10 X64. The commercial solver is with the default parameters set.

To give an example of the computational result, the disposition timetable is obtained in case of ('84119','YC',(dep,2700)), shown as Figure 8. Here the train "84119" is delayed departure from the " $Y C$ " station, and the primary delay is $2,760 \mathrm{~s}$. The disrupted situation is presented by the red circle in the Figure 8 . The obtained result shows that the total delays of trains at their destinations are 2,326s. Because of the large primary departure delays at the " $Y C$ " station, the order of occupation of "YC-JY" section is changed. The headway of the adjacent trains follows the minimum headway time. From the obtained disposition timetable, the total number of influenced trains is two. Moreover, the recovery time of rescheduled timetable is $06: 44$, that is, the train can be departed from the original station at the scheduled departure time.

The computational results obtained are reported in Table 4 when the different disrupted scenarios are considered. In the Table 4, the first column denotes the disruption scenario which is considered in the proposed model. In the experiment, we consider two objective functions, respectively. The considered objective functions are as follows:

(1) Obj-1: The total final delays which are the total arrival delays of all trains arriving at their destination.

(2) Obj-2: The consecutive delays which are the total arrival and departure delays of all trains at their stations.

The values of two objective functions are reported at the two and three columns, respectively. Moreover, the four and five columns denote the computational times, and the last column reports the gap of feasible solution to optimal solution. The results show that all disruption instances are solved within 10 s optimally.

Figure 9 shows that the values of objective functions increase as the primary departure delays increase, and the values of objective functions do not increase when the primary departure delays increase to a certain point (the primary departure delays is 2,700s).

Figure 8.

Disposition timetable in case of ('84119',' YC', (dep, 2700))

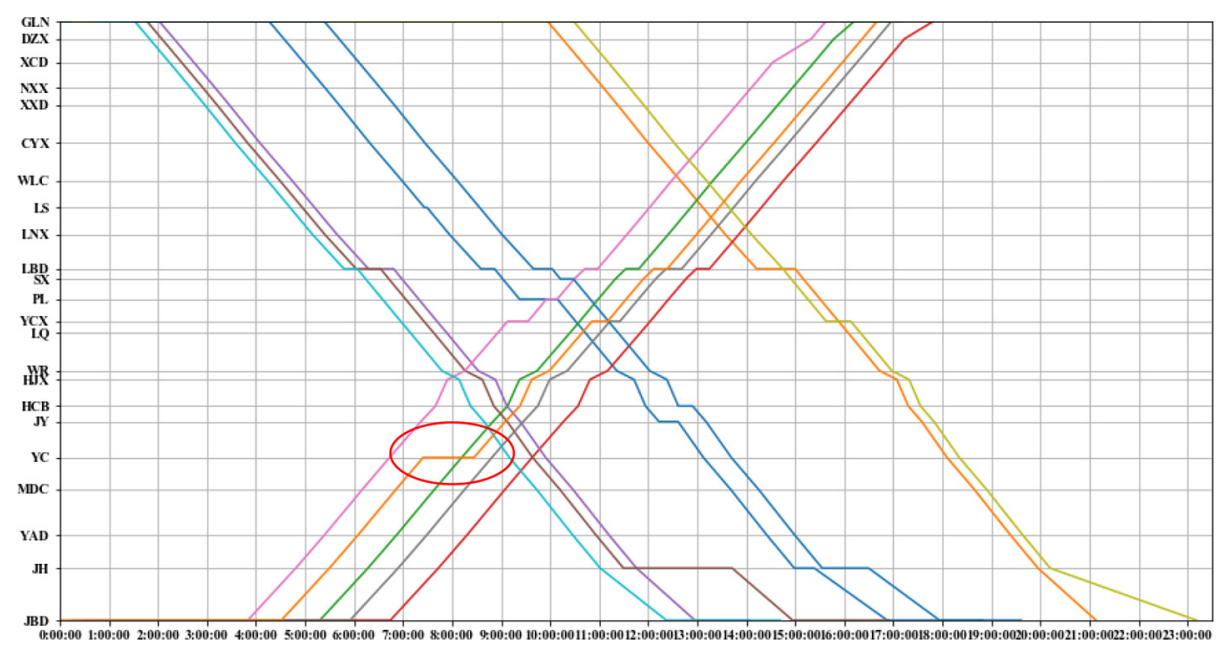




\begin{tabular}{|c|c|c|c|c|c|c|c|}
\hline \multirow[b]{2}{*}{ Index } & \multirow[b]{2}{*}{ Instance } & \multicolumn{2}{|c|}{ Objective function (s) } & \multicolumn{2}{|c|}{ Computational time (s) } & \multirow[b]{2}{*}{ Gap (\%) } & \multirow{2}{*}{$\begin{array}{l}\text { Freight train } \\
\text { dispatching }\end{array}$} \\
\hline & & Obj-1 & Obj-2 & Obj-1 & Obj-2 & & \\
\hline 1 & ('tr','YY',(dep,1800)) & 1220 & 242389 & 2 & 4 & 0 & \\
\hline 2 & ('tr','YC',(dep,2100)) & 1508 & 252562 & 3 & 5 & 0 & \\
\hline 3 & ('tr','YC',(dep,2400)) & 1887 & 271724 & 3 & 5 & 0 & \\
\hline 4 & ('tr','YY',(dep,2700)) & 2326 & 296044 & 4 & 6 & 0 & \\
\hline 5 & ('tr','YC',(dep,3000)) & 2326 & 298240 & 3 & 5 & 0 & 97 \\
\hline 6 & ('tr','YC',(dep,3300)) & 2326 & 298240 & 4 & 5 & 0 & \\
\hline 7 & ('tr','YC',(dep,3600)) & 2326 & 298240 & 5 & 6 & 0 & \\
\hline 8 & $\begin{array}{l}\text { ('tr','YC',(dep,3600)) } \\
(\text { 'tr','MD', }(d e p, 3600))\end{array}$ & 5924 & 436105 & 9 & 9 & 0 & \\
\hline 9 & $\begin{array}{l}\text { ('tr','YC',(dep,3600)) } \\
\text { ('tr','YAD',(dep,3600)) }\end{array}$ & 5924 & 442435 & 10 & 10 & 0 & Table 4. \\
\hline 10 & $\begin{array}{l}\text { ('tr','YC',(dep,3600)) } \\
\text { ('tr','JY',(dep,3600)) }\end{array}$ & 5924 & 423445 & 10 & 10 & 0 & $\begin{array}{l}\text { Results for various } \\
\text { disrupted scenarios }\end{array}$ \\
\hline 11 & $\begin{array}{l}\text { ('tr','YC',(dep,3600)) } \\
(\text { 'tr','HCB',(dep,3600)) }\end{array}$ & 5924 & 416551 & 12 & 11 & 0 & $\begin{array}{l}\text { obtained by the } \\
\text { proposed method }\end{array}$ \\
\hline
\end{tabular}

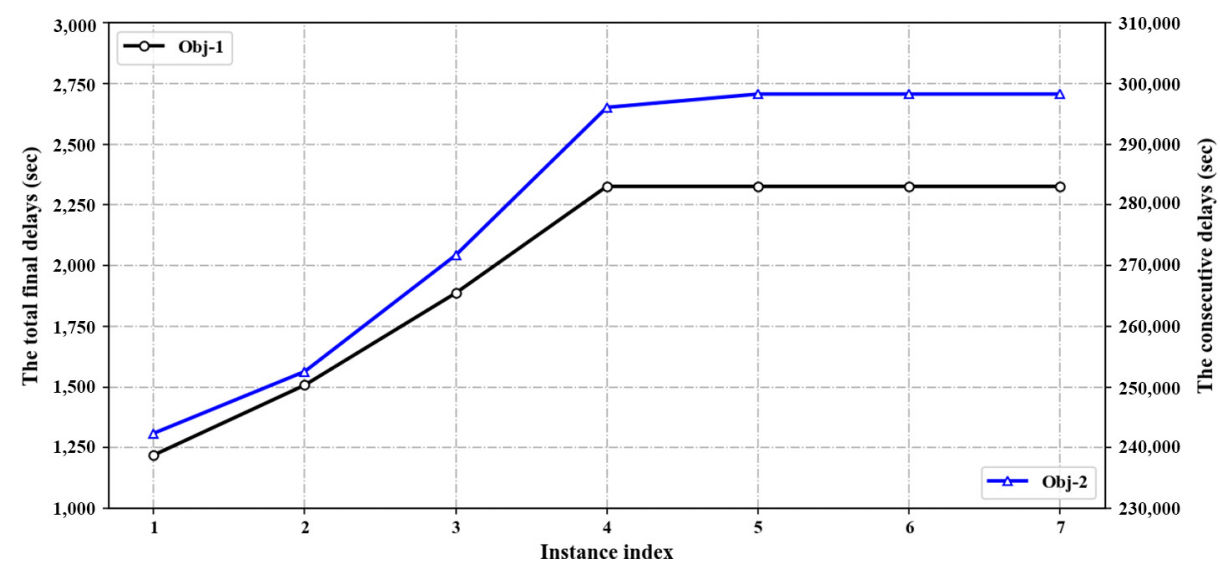

Figure 9.

Values of two objective functions with different disruption instances

Moreover, the consecutive delays of each disrupted instance are more than its total final delays obviously.

\section{Conclusion and further work}

In this paper we studied the real-time rescheduling problem for the freight heavy haul railway in case of disrupted situations. An integer linear programming model based on alternative graph is formulated; therefore, the capacity of the railway stations is considered in the proposed model. A real-world experiment of the Haolebaoji-Ji'an freight heavy haul railway line is implemented to test our model. The optimum disposition timetables are generated in real-time for all disrupted instances.

In our paper, the freight heavy haul railway line is divided into block sections. The parameters of the freight heavy haul railway are analyzed based on the large volume of history actual operation data. The route of train is scheduled and cannot be changed. The real-time train dispatching measures, such as re-timing and re-ordering are applied to restore the disrupted situations. The two objective functions: 
(1) the total final delays; and

(2) the consecutive delays are considered in the proposed model.

The results give insight into that the consecutive delays are more than the total final delays, and moreover, the obtained disposition timetable is also different when the same disruption occurs. Furthermore, the total final delays or consecutive delays increase as the primary delays increase.

Several further research directions are available. First, in this paper, we only consider double-track segments; however, there exist single-track segments in the freight haul railway system. Thus, the real-time freight heavy haul railway traffic management model extending our proposed model adapting the single-track segment constraints. Second, an efficient algorithm should be developed to satisfy the real-time requirement when more trains are operated in the future. Finally, the freight vehicle turnaround constraints should be taken into account for the freight heavy haul trains dispatching problem.

\section{References}

Binder, S., Maknoon, Y. and Bierlaire, M. (2017), "The multi-objective railway timetable rescheduling problem", Transportation Research Part C: Emerging Technologies, Vol. 78, pp. 78-94, doi: 10.1016/j.trc.2017.02.001.

Cacchiani, V., Huisman, D., Kidd, M., Kroon, L., Toth, P., Veelenturf, L. and Wagenaar, J. (2014), “An overview of recovery models and algorithms for real-time railway rescheduling", Transportation Research Part B: Methodological, Vol. 63, pp. 15-37, doi: 10.1016/j.trb.2014.01.009.

Cordeau, J.F., Toth, P. and Vigo, D. (1998), "A survey of optimization models for train routing and scheduling”, Transportation Science, Vol. 32 No. 4, pp. 380-404, doi: 10.1287/trsc.32.4.380.

Corman, F. and Meng, L. (2015), "A review of online dynamic models and algorithms for railway traffic management”, IEEE Transactions on Intelligent Transportation Systems, Vol. 16 No. 3, pp. 1274-1284, doi: 10.1109/TITS.2014.2358392.

Corman, F., D'Ariano, A., Hansen, I.A. and Pacciarelli, D. (2011), "Optimal multi-class rescheduling of railway traffic", Journal of Rail Transport Planning and Management, Vol. 1 No. 1, pp. 14-24, doi: 10.1016/j.jrtpm.2011.06.001.

Corman, F., D’Ariano, A., Pacciarelli, D. and Pranzo, M. (2010), "A tabu search algorithm for rerouting trains during rail operations", Transportation Research Part B: Methodological, Vol. 44 No. 1, pp. 175-192.

Corman, F., D’Ariano, A., Marra, A.D., Pacciarelli, D. and Samà, M. (2017), "Integrating train scheduling and delay management in real-time railway traffic control", Transportation Research Part E: Logistics and Transportation Review, Vol. 105, pp. 213-239, doi: 10.1016/j.tre.2016.04.007.

D'Ariano, A. and Pranzo, M. (2009), "An advanced real-time train dispatching system for minimizing the propagation of delays in a dispatching area under severe disturbances", Networks and Spatial Economics, Vol. 9 No. 1, pp. 63-84, doi: 10.1007/s11067-008-9088-1.

D'Ariano, A., Pacciarelli, D. and Pranzo, M. (2007a), "A branch and bound algorithm for scheduling trains in a railway network", European Journal of Operational Research, Vol. 183 No. 2, pp. 643-657, doi: 10.1016/j.ejor.2006.10.034.

D'Ariano, A., Pacciarelli, D. and Pranzo, M. (2008), "Assessment of flexible timetables in real-time traffic management of a railway bottleneck", Transportation Research Part C: Emerging Technologies, Vol. 16 No. 2, pp. 232-245, doi: 10.1016/j.trc.2007.07.006.

D’Ariano, A., Pranzo, M. and Hansen, I.A. (2007b), "Conflict resolution and train speed coordination for solving real-time timetable perturbations", IEEE Transactions on Intelligent Transportation Systems, Vol. 8 No. 2, pp. 208-222, doi: 10.1109/TITS.2006.888605. 
Dollevoet, T., Huisman, D., Schmidt, M. and Schöbel, A. (2012), "Delay management with rerouting of passengers", Transportation Science, Vol. 46 No. 1, pp. 74-89, doi: 10.1287/trsc.1110.0375.

Dollevoet, T., Huisman, D., Kroon, L., Schmidt, M. and Schöbel, A. (2015), "Delay management including capacities of stations”, Transportation Science, Vol. 49 No. 2, pp. 185-203, doi: 10.1287/ trsc.2013.0506.

Lusby, R.M., Haahr, J.T., Larsen, J. and Pisinger, D. (2017), “A branch-and-price algorithm for railway rolling stock rescheduling", Transportation Research Part B: Methodological, Vol. 99, pp. 228-250, doi: 10.1016/j.trb.2017.03.003.

Potthoff, D., Huisman, D. and Desaulniers, G. (2010), "Column generation with dynamic duty selection for railway crew rescheduling”, Transportation Science, Vol. 44 No. 4, pp. 493-505, doi: 10.1287/ trsc. 1100.0322 .

Pranzo, M. (2004), “A real-time train dispatching system based on the blocking time theory, in: a world of transport, infrastructure and logistics: proceedings; 8th international TRAIL congress 2004", Selected Papers, pp. 129-152.

Quaglietta, E., Pellegrini, P., Goverde, R.M.P., Albrecht, T., Jaekel, B., Marlière, G., Rodriguez, J., Dollevoet, T., Ambrogio, B., Carcasole, D., Giaroli, M. and Nicholson, G. (2016), "The on-TIME real-time railway traffic management framework: a proof-of-concept using a scalable standardised data communication architecture", Transportation Research Part C: Emerging Technologies, Vol. 63, pp. 23-50, doi: 10.1016/j.trc.2015.11.014.

Veelenturf, L.P., Kidd, M.P., Cacchiani, V., Kroon, L.G. and Toth, P. (2016), “A railway timetable rescheduling approach for handling large-scale disruptions", Transportation Science, Vol. 50 No. 3, pp. 841-862, doi: 10.1287/trsc.2015.0618.

Zilko, A.A., Kurowicka, D. and Goverde, R.M.P. (2016), "Modeling railway disruption lengths with Copula Bayesian networks", Transportation Research Part C: Emerging Technologies, Vol. 68, pp. 350-368, doi: 10.1016/j.trc.2016.04.018.

\section{Corresponding author}

Mingming Wang can be contacted at: 17114240@bjtu.edu.cn
Freight train dispatching approach

For instructions on how to order reprints of this article, please visit our website: 